\title{
MODELLING HIGH TEMPERATURE ROLLING TEXTURES OF FCC METALS
}

\author{
Cl. MAURICE, $\dagger$ J. H. DRIVER, $\ddagger$ and L. S. TÓTH§ \\ †CRV SA, BP 27, 38340 Voreppe, France \\ $\$$ Ecole des Mines, Département Matériaux, 158 Cours Fauriel, \\ 42023 Saint-Etienne Cedex 2, France \\ §Institute for General Physics, Eötvös University, 1445. Budapest, P.O.B. 323, \\ Hungary
}

(12 March 1992)

\begin{abstract}
A partially relaxed constraints grain deformation model is proposed to explain the influence of temperature on the rolling textures of fcc metals. The effects of the grain plastic shear in the TD/RD plane and the role of the rate sensitivity of crystallographic slip on the evolution of the texture have been investigated by numerical simulations for a random initial texture. The rate sensitivity and the TD/RD shear are assumed to increase with temperature. The progression from the Copper $\{112\}\langle 111\rangle$ component towards Brass $\{110\}\langle 112\rangle$ and $S\{123\}\langle 634\rangle$ type textures is predicted at higher values of the rate sensitivity and the TD/RD shear. These model predictions compare well with published hot rolling textures of aluminium alloys. The concept of grain shear partial relaxation has been validated by room and high temperature channel die tests on $\{110\}\langle 112\rangle$ oriented Al crystals constrained between aluminium polycrystals.
\end{abstract}

KEY WORDS Rate sensitive model, partially relaxed, channel die experiments.

\section{INTRODUCTION}

The typical evolution of deformation textures during the cold rolling of medium to high stacking fault energy fcc metals can be adequately described by classical Taylor-type models, i.e. the Full Constraints (FC) or Relaxed Constraints (RC) models, see for example the reviews by Van Houtte (1986) and Hirsch and Lücke (1988). These models can be extended to low stacking fault energy alloys, or equivalently to low temperature deformation, by allowing for twinning in addition to the usual $\{111\}\langle 110\rangle$ slip modes. Twinning promotes the Brass (Bs) $\{110\}\langle 112\rangle$ component at the expense of the other usual rolling texture components $S\{123\}\langle 634\rangle$ and $\mathrm{Cu}\{112\}\langle 111\rangle$.

However, it is well known that high temperature rolling in the homologous temperature range $0.4-0.8 T_{m}$, (and when dynamic recrystallization does not occur) also leads to the development of texture components which can be significantly different from those developed by cold rolling. In particular, hot rolling often develops a strong Bs component which, at these temperatures, cannot be ascribed to twinning. A common example is the strong Bs component of aluminium alloys rolled in the temperature range $300-500^{\circ} \mathrm{C}$. The origin of this high temperature texture component has been discussed in detail by Bacroix and Jonas, (1988a). The most frequent explanations are (i) non-octohedral slip 
(Bacroix and Jonas, 1988b) and (ii) a Sachs type RC model in which the grain TD/RD shear is completely relaxed (Hirsch and Lücke, 1988). Neither explanation is totally free from criticism. Non-octahedral slip has been observed at low strain rates, $<10^{-3} \mathrm{~s}^{-1}$, but not, as yet, at the high strain rates, $>10 \mathrm{~s}^{-1}$, typical of industrial hot rolling schedules. Secondly, it is generally agreed that the Sachs type grain relaxation leads to unreasonably high strain rate incompatibilities between grains.

These physical problems have hindered texture modelling for hot rolling, one of the most technologically important metallurgical processes. The absence of adequate high temperature deformation texture predictions also limits the usefulness of texture modelling during subsequent treatments, such as cold rolling or recrystallization, which depend upon the metallurgical state of the hot deformed material.

It is the purpose of this paper to present a simple variation of the $\mathrm{RC}$ model that can be used to quantitatively predict the development of hot rolling textures. The model is applied to the calculation of ODFs (orientation distribution functions) of hot rolled fcc metals as a function of strain. The results are compared with the textures of hot rolled $\mathrm{Al}$ alloys taken from the literature.

\section{THE PARTIALLY RELAXED CONSTRAINTS MODEL}

\section{Grain Shear Strains}

As mentioned above, a strong Bs rolling texture component is predicted by a Taylor type model in which the TD/RD shear is completely relaxed (this is the $\dot{\varepsilon}_{12}$ shear strain rate in a reference frame for which $X_{1}=\mathrm{RD}, X_{2}=\mathrm{TD}$ and $\left.X_{3}=\mathrm{ND}\right)$. However, during rolling the grain shape tends towards a "surf board" shape which means that a totally free $\dot{\varepsilon}_{12}$ shear rate would involve large intergranular strain rate incompatibilities, extending over relatively large volumes of the grains. The high reaction stresses developed in the surrounding grains would therefore strongly reduce the $\dot{\varepsilon}_{12}$ shear rate. In fact a totally free $\dot{\varepsilon}_{12}$ shear strain rate is associated with a zero shear stress $\sigma_{12}$, as, for example, in channel die compression of a single crystal. Under these conditions $\dot{\varepsilon}_{12}$ takes a value, denoted $\dot{\varepsilon}_{12}^{0}$ which is, of course, orientation dependent, being, for example, very high for the Bs orientation $(0.35 \dot{\varepsilon})$ and zero for the Goss orientation $\{011\}\langle 100\rangle$.

It is physically more realistic to allow the grains to undergo small $\dot{\varepsilon}_{12}$ strain rates, significantly less than $\dot{\varepsilon}_{12}^{0}$. There is some experimental evidence for this from channel die experiments on Al polycrystals, (Fortunier (1987) and Mizera (1988)). Recent finite element calculations of grain deformations in a polycrystal undergoing plane strain compression, (Becker and Lalli (1991)), also reveal $\varepsilon_{12}$ grain shears, particularly for grains near to the Bs orientation. Experimental results described below on partially constrained crystals illustrate this effect quite clearly.

It is therefore proposed that during high temperature rolling individual grains undergo a shear strain rate $\dot{\varepsilon}_{12}$ reduced by a factor $\zeta$ compared with the RC value $\dot{\varepsilon}_{12}^{0}$, where $0<\zeta<1$. For most conditions, $\zeta$ is of the order of 0.2 so that the $\varepsilon_{12}$ shears are relatively small. 
At the same time, in conformity with the usual RC lath model, the $\varepsilon_{13}$ shear $\left(\varepsilon_{\mathrm{RD} / \mathrm{ND}}\right)$ is taken at the value $\varepsilon_{13}^{0}$ corresponding to $\sigma_{13}=0$.

The stress and strain boundary conditions imposed on the grains in the present model are therefore

$$
\boldsymbol{\sigma}=\left(\begin{array}{ccc}
\sigma_{11} & \sigma_{12} & 0 \\
\sigma_{12} & \sigma_{22} & \sigma_{23} \\
0 & \sigma_{23} & \sigma_{33}
\end{array}\right) \quad \dot{\varepsilon}=\left(\begin{array}{ccc}
\dot{\varepsilon} & \zeta \dot{\varepsilon}_{12}^{0} & \dot{\varepsilon}_{13}^{0} \\
\zeta \dot{\varepsilon}_{12}^{0} & 0 & 0 \\
\dot{\varepsilon}_{13}^{0} & 0 & -\dot{\varepsilon}
\end{array}\right)
$$

\section{The Rate Sensitivity Model}

With increasing temperatures the flow stresses of metallic materials become increasingly sensitive to the deformation rate. High temperature deformation and texture evolution can then be reasonably treated by a rate sensitive model.

The model used here is that developed by Tóth, Gilormini and Jonas (1988) which has been applied to the cases of torsion texture development (Tóth et al. (1988)) and single crystal channel die deformation (Tóth, Jonas and Neale (1990)). Following Pierce, Asaro and Needleman (1983), the rate sensitivity of slip is modelled by a power law relation between the shear rate $\dot{\gamma}^{s}$ and the resolved shear stress $\tau^{s}$

$$
\tau^{s}=\tau_{s}^{0} \operatorname{sgn}\left(\dot{\gamma}_{s}\right)\left|\frac{\dot{\gamma}^{s}}{\dot{\gamma}_{0}}\right|^{m}
$$

$m$ is the slip system rate sensitivity $(m \geq 0), \tau_{s}^{0}$ and $\dot{\gamma}_{0}$ are reference quantities for the shear stresses and the slip rates. For the case of isotropic hardening considered here $\tau_{s}^{0}=\tau_{0}$.

Equation (1) implies the existence of a stress potential which is rounded, strictly convex and tends to the Bishop and Hill yield surface when $\tau_{0}$ is set equal to $\tau_{c}$ the critical resolved shear stress for rate insensitive slip, and $m$ tends to zero. The stress response of a grain to imposed strain rates $\dot{\varepsilon}_{i j}$ is obtained by solving the following system of non-linear equations

$$
\dot{\varepsilon}_{i j}=\frac{\dot{\gamma}_{0}}{2 \tau_{0}^{1 / m}} \sum_{s}\left(m_{i j}^{s}+m_{j i}^{s}\right) m_{k l}^{s} \sigma_{k l}\left|m_{m n}^{s} \sigma_{m n}\right|^{(1 / m-1)}
$$

where the quantities $m_{i j}^{s}$ are defined by the slip plane normal $n^{s}$ and the slip direction $b^{s}$ by $m_{i j}^{s}=b_{i}^{s} n_{j}^{s}$.

The number of unknowns in Eq. (2) (the components of $\sigma$ ) usually equals the number of imposed independent strain rate components, i.e. 5 for the fully constrained deformation mode.

In the partially relaxed constrained mode described above, the 3 fully constrained strain rate components $\dot{\varepsilon}_{11}, \dot{\varepsilon}_{33}$ and $\dot{\varepsilon}_{23}$ are first used to calculate an initial stress state with 3 unknowns, $\sigma_{11}, \sigma_{33}$ and $\sigma_{23}\left(\sigma_{12}, \sigma_{13}=0\right)$. The relaxed constraints values of the non-prescribed strain rate components $\dot{\varepsilon}_{12}^{0}$ and $\dot{\varepsilon}_{13}^{0}$ are then obtained directly from Eq. (2) using this 3 component stress state.

The stress state corresponding to the boundary conditions for the partially relaxed grain deformation mode $\left(\dot{\varepsilon}_{12}=\zeta \dot{\varepsilon}_{12}^{0}\right)$ is then recalculated from Eq. (2) with 4 unknown stress components $\left(\sigma_{11}, \sigma_{33}, \sigma_{23}\right.$ and $\left.\sigma_{12}\right)$. This value of $\sigma$ is then 
used to evaluate the resolved shear stress via the usual relation

$$
\tau_{s}=m_{i j}^{s} \sigma_{i j}
$$

The slip rates $\dot{\gamma}^{s}$ are then obtained directly from Eq. (1). It is evident from this equation and the existence of a rounded yield potential that rate sensitive slip always leads to a unique slip rate distribution as well as to a unique stress state.

Finally the lattice rotation rate $\dot{\boldsymbol{\Omega}}$ is obtained from

$$
\dot{\Omega}_{i j}=\dot{d}_{i j}-\sum_{s} m_{i j}^{s} \dot{\gamma}^{s}
$$

where $\dot{d}_{i j}$ is the displacement gradient velocity tensor of the material with respect to the laboratory. For the grain deformation modes considered here, the components of $\dot{\mathbf{d}}$ that take zero values can be used to define $\dot{\boldsymbol{\Omega}}$ as follows:

$$
\begin{aligned}
& \dot{\Omega}_{12}=\sum_{s} m_{21}^{s} \dot{\gamma}^{s} \\
& \dot{\Omega}_{13}=\sum_{s} m_{31}^{s} \dot{\gamma}^{s} \\
& \dot{\Omega}_{23}=\sum_{s} m_{32}^{s} \dot{\gamma}^{s}
\end{aligned}
$$

\section{EXPERIMENTAL}

Channel die plane strain compression tests on partially constrained $\mathrm{Al}$ single crystals have been carried out to measure the amount of $\dot{\varepsilon}_{12}$ shears at different temperatures.

High purity aluminium single crystals with a $\{110\}\langle 112\rangle$ orientation were electron beam welded to aluminium polycrystalline blocks. As shown in Figure 1 the volumes of the central crystal and the surrounding polycrystalline material are similar. The $5 \times 5 \times 28 \mathrm{~mm}$ samples were electropolished and a reference grid was

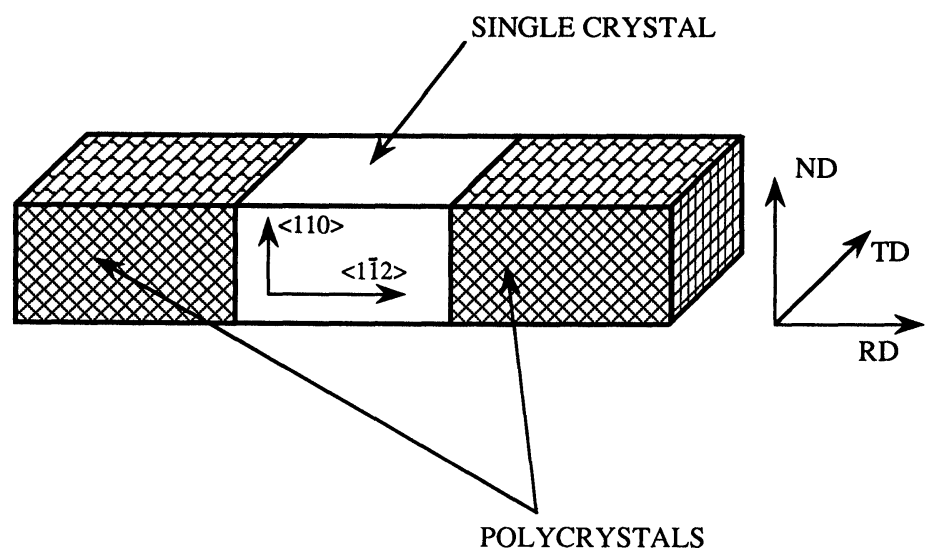

Figure $1\{110\}\langle 112\rangle$ oriented $\mathrm{Al}$ single crystal welded to Al polycrystalline ends. 
inscribed with a $1 \mathrm{~mm}$ spacing on a compression and a transverse face to measure the shear strains and the degree of deformation homogeneity.

These "constrained" crystals were deformed to compressive true strains of about 0.6 at temperatures of $22^{\circ} \mathrm{C}$ and $350^{\circ} \mathrm{C}$. A standard teflon lubricated punch and die apparatus was used at room temperature (Orlans-Joliet B. et al. (1990)). High temperature tests were carried out in a suitably modified channel die using Teflon and graphite lubrication at a strain rate of $10^{-2} \mathrm{~s}^{-1}$. Previous tests have shown that homogeneous uniform deformations up to strains of 1.5 can be achieved, up to $400^{\circ} \mathrm{C}$, with this equipment (Maurice (1992)).

Figures 2(a) and (b) show the grid displacements on the compression face due to the $\varepsilon_{12}$ shears after true strains of 0.59 at $22^{\circ} \mathrm{C} \mathrm{(a)} \mathrm{and} 0.53$ at $350^{\circ} \mathrm{C}$ (b). The important feature illustrated by these figures is the shear strain that occurs in the central $\{110\}\langle 112\rangle$ crystal despite the presence of the polycrystalline ends. The latter partially constrain the crystal since the average measured shear strains are roughly half the theoretical value for unconstrained shear $\left(\dot{\varepsilon}_{12}^{0}=(\sqrt{ } 2 / 4) \dot{\varepsilon}\right)$. The measured shears are in fact 0.14 at room temperature (i.e. $49 \%$ of the theoretical finite shear corresponding to a compressive true strain of 0.59 ) and 0.11 at $350^{\circ} \mathrm{C}$ (i.e. $42 \%$ of the theoretical value obtained for a compressive true strain of 0.53 ).

These values indicate apparently similar behaviour at room and high temperature. However, close inspection of the reference grids on the polycrystals indicate significant differences: at room temperature, the polycrystals are sheared by the

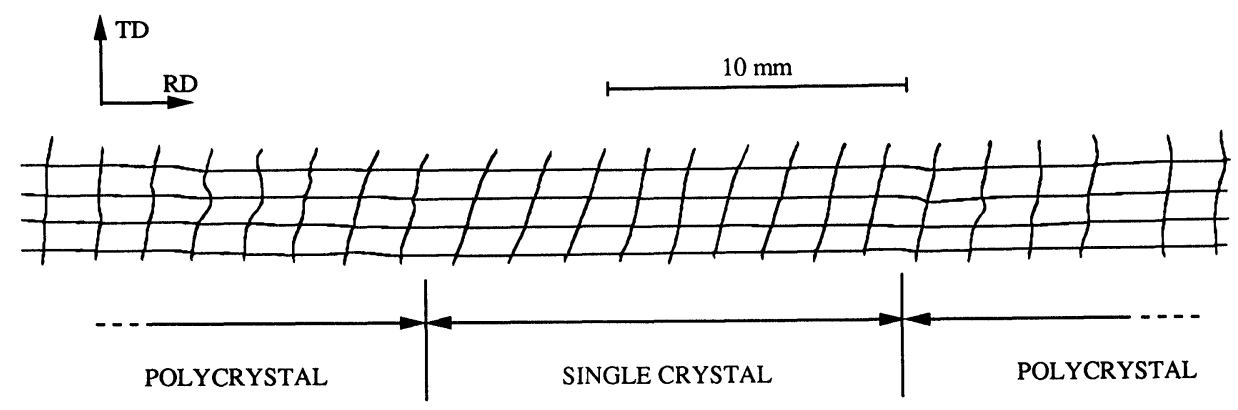

(a)

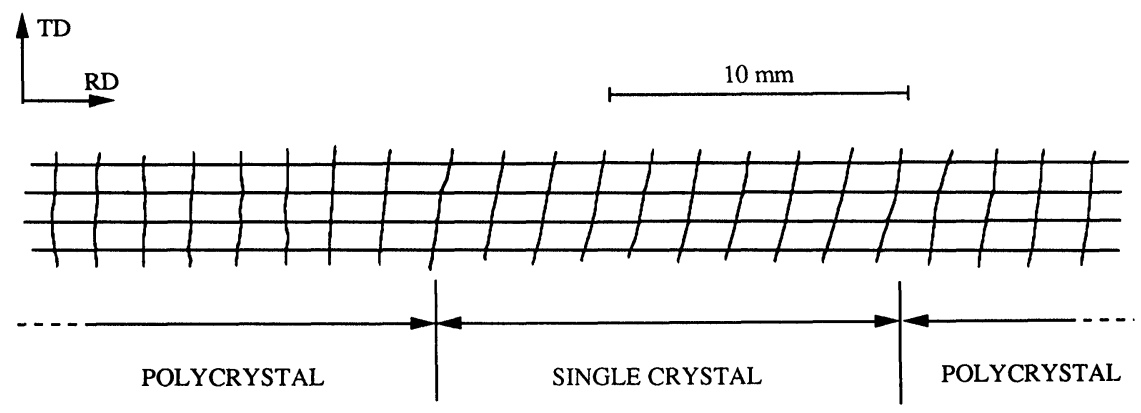

(b)

Figure 2 Reference grids inscribed on the compression face after deformation (a) at $22^{\circ} \mathrm{C}$ up to a strain of 0.59 , (b) at $350^{\circ} \mathrm{C}$ up to a strain of 0.53 . 
central crystal for distances over half their length, whereas at high temperature the $\varepsilon_{12}$ shear of the polycrystals is negligible, implying large shear strain rate gradients at the poly/monocrystal interface. This suggests that at high temperature shear strain gradients between neighbouring grains can be accommodated relatively easily. Under the more typical rolling conditions of a small grain constrained by similar sized grains, one can therefore expect easier accommodation of the grain shear strains at high temperature, i.e. the individual grains can shear more easily leading to higher $\zeta$ values.

\section{NUMERICAL SIMULATION PROCEDURE}

Most calculations were performed with a set of 936 initial grain orientations distributed in Euler space so that the value of the orientation distribution function $f(g)=1 \pm 0.03$ (Fortunier (1987)). The numerical simulations using these orientations then describe the theoretical texture evolution from a set of initial "random" orientations.

For each grain orientation, the lattice rotations and the new orientations were calculated by the method described in Section 2, using strain increments of 0.025 for finite strains up to 3 . At fixed strains, the file of discrete orientations was converted into ODFs using the Gaussian function method with a spread of $5^{\circ}$. ODFs were printed out as such, or to facilitate quantitative comparisons, the orientation densities $f(g)$ were evaluated along standard skeleton lines such as the $\alpha$ and $\beta$ fibres.

Two parameters have been used in the simulations to characterize the influence of temperature; the strain rate sensitivity $m$ and the $\dot{\varepsilon}_{12}$ shear rate parameter $\zeta$. It is well known that $m$ increases with temperature. Tegart (1966) quotes values for aluminium from $0.02\left(20^{\circ} \mathrm{C}\right)$ to $0.07\left(300^{\circ} \mathrm{C}\right)$ and $0.15\left(500^{\circ} \mathrm{C}\right)$. More recent data indicate somewhat higher values; 0.23 for a $1100 \mathrm{Al}$ alloy measured by Brown, $\mathrm{Kim}$ and Anand (1989) in the range $300-500^{\circ} \mathrm{C}$ and 0.25 to 0.29 for $\mathrm{Al}-2 \% \mathrm{Mg}$ (Chen and Kocks (1991)) at $300-400^{\circ} \mathrm{C}$. We have used a range of $m$ values from 0.05 to 0.25 as being typical of the behaviour of most $\mathrm{Al}$ alloys in the temperature range $20^{\circ} \mathrm{C}-500^{\circ} \mathrm{C}$.

The variation of $\zeta$ with temperature during rolling is scarcely known at all. Our experiments, for the particular case of a partially constrained crystal in channel die compression, indicate $\zeta$ values of about 0.5 . However, we strongly suspect that for rolling of small grains, such large values can only be achieved at high temperatures. At low temperatures the intergranular strain rate incompatibilities should substantially reduce $\zeta$. Values used here vary systematically from 0 to 0.5 which can be considered to represent extremes of material behaviour.

The entire set of values of the parameters used in the simulations is given below

$$
\begin{array}{llllll}
m: 0.05, & 0.1, & 0.15, & 0.2, & 0.25 \\
\zeta: 0, & 0.1, & 0.25, & 0.5 & & \\
\varepsilon: 0.5, & 1, & 1.5, & 2, & 2.5, & 3
\end{array}
$$


To simplify the interpretation, results will be described in detail for 3 conditions which can be considered to qualitatively represent 3 temperature regimes.
(i) low temperature
$\left(T<0.4 T_{m}\right)$
$m=0.05, \quad \xi=0.1$
(ii) medium temperature
$\left(0.4 T_{m}<T<0.7 T_{m}\right)$
$m=0.15, \quad \zeta=0.25$
(iii) high temperature
$\left(T>0.7 T_{m}\right)$
$m=0.2, \quad \zeta=0.5$

\section{RESULTS}

The ODFs obtained by the numerical simulations using the above 3 conditions are shown in Figure $3(a, b, c)$, at a true strain of 2 . The usual rolling texture components $\mathrm{Cu}\{112\}\langle 111\rangle, \mathrm{S}\{123\}\langle 634\rangle$ and $\mathrm{Bs}\{110\}\langle 112\rangle$ are developed along the $\beta$ fibre together with some Goss on the $\alpha$ fibre. The important feature of these simulated rolling textures is the change in the relative intensity of these texture components as a function of the parameters $m$ and $\zeta$. Figure 3(a) illustrates a typical low temperature rolling texture with a maximum intensity at the $\mathrm{Cu}\{112\}\langle 111\rangle$ orientation together with a strong $\mathrm{S}$ and a weaker Bs component.

In the intermediate condition, Figure $3(\mathrm{~b})$, both the $\mathrm{Bs}$ and $\mathrm{S}$ densities increase substantially so that $S$ becomes the strongest component, and finally as shown in Figure 3(c) in the "high" temperature condition the maximum intensity occurs near the Bs orientation. These results are presented quantitatively as $f(g)$ plots along the $\beta$ fibre for different rolling strains in Figures 4(a), (b), (c). It is clear that the model reproduces the essential features of both cold and hot rolling fcc texture development, i.e. a strong $\mathrm{Cu}$ component at low $m$ and $\zeta$ and a strong Bs component at high $m$ and $\zeta$.

Influence of $m$ and $\zeta$ Figure 5 illustrates the influence of the $\zeta$ parameter on the $\beta$ fibre texture components at a constant strain rate sensitivity of $m=0.05$. At $\zeta=0$ a strong $\mathrm{Cu}$ component is developed as expected from the classical relaxed constraints model with a free $\varepsilon_{13}$ shear. At higher $\zeta$ values, however, it is the $S$ component that increases most and dominates for $\zeta \geq 0.25$. To the authors' knowledge, the role of a partial relaxation of $\dot{\varepsilon}_{12}$ in strengthening the $S$ component has not been previously considered. For example, Hirsch and Lücke (1988) have shown that total relaxation of $\dot{\varepsilon}_{12}(\xi=1)$ virtually eliminates the $S$ component at the expense of Bs. They argue that the $\dot{\varepsilon}_{23}$ shear is necessary to stabilise the $S$ component. The present results show that a partially relaxed $\dot{\varepsilon}_{12}$ shear can also stabilise $S$.

Figure 5(c) also shows that at $\zeta=0.5$ the Bs component can become stronger than $\mathrm{Cu}$. However if $\zeta<1$ very strong Bs components can only be achieved with a higher $m$ value. This is demonstrated in Figure 6 by the $\beta$ fibre plots at constant $\zeta=0.25$ and increasing $m$. As the rate sensitivity increases, the $S$ component decreases in intensity, while Bs increases. Finally if $\zeta=0$, corresponding to the more conventional Taylor models, then increasing $\boldsymbol{m}$ results in a slightly weaker $\mathrm{Cu}$ and a somewhat stronger Bs components. This effect has also been pointed out by Chen and Kocks (1991). 


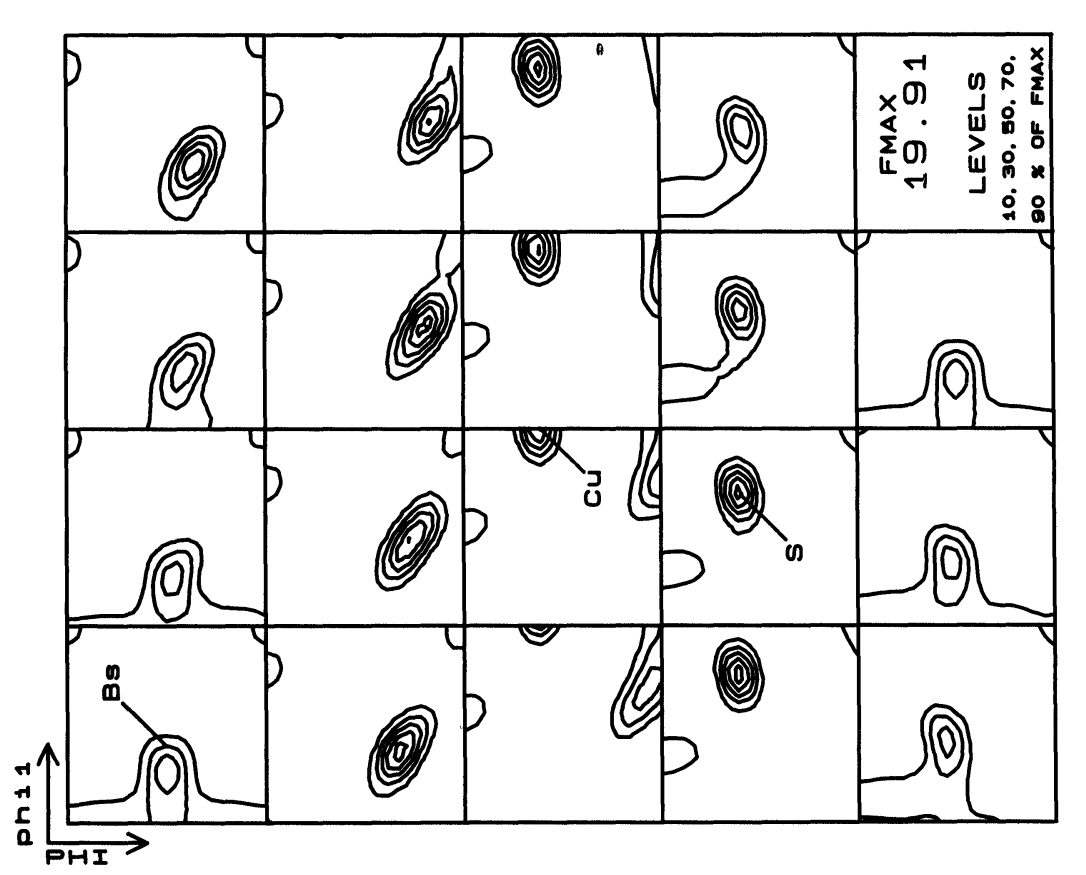

20

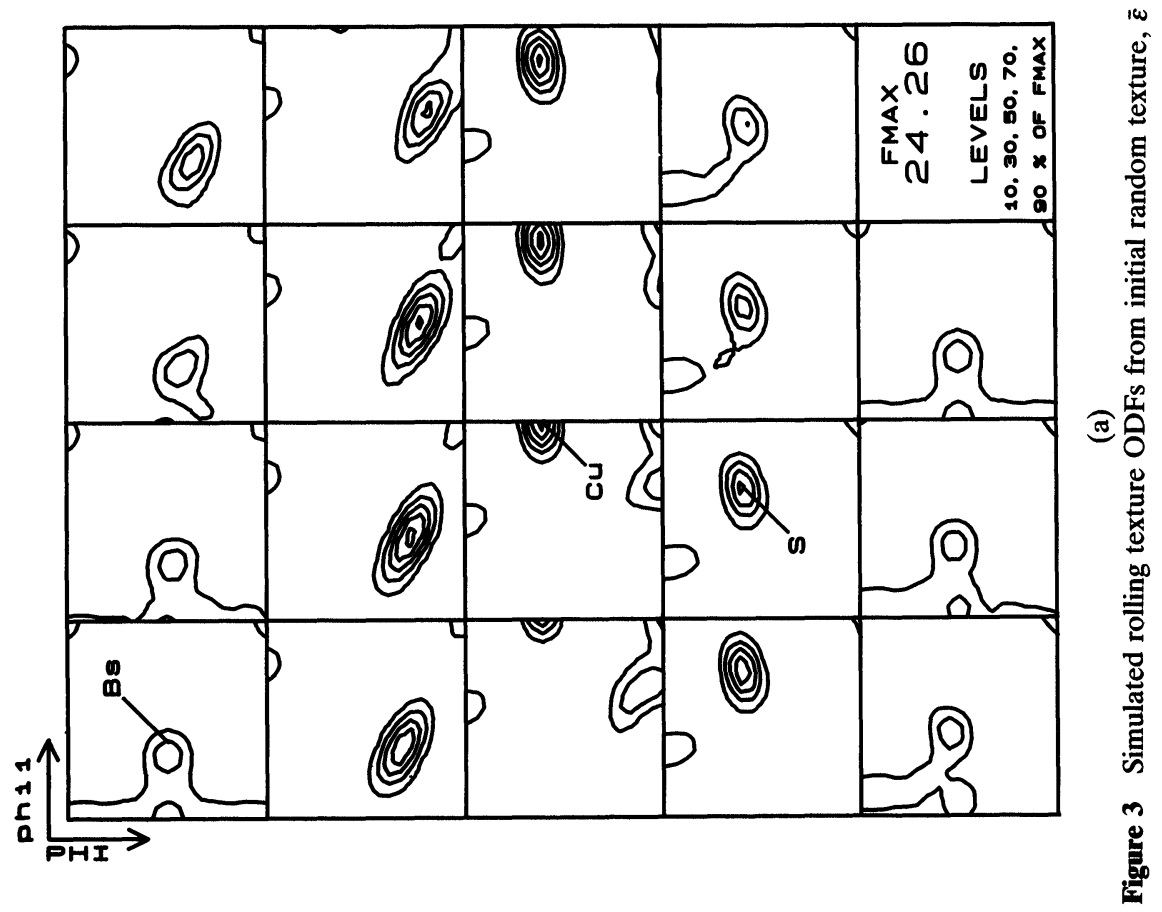




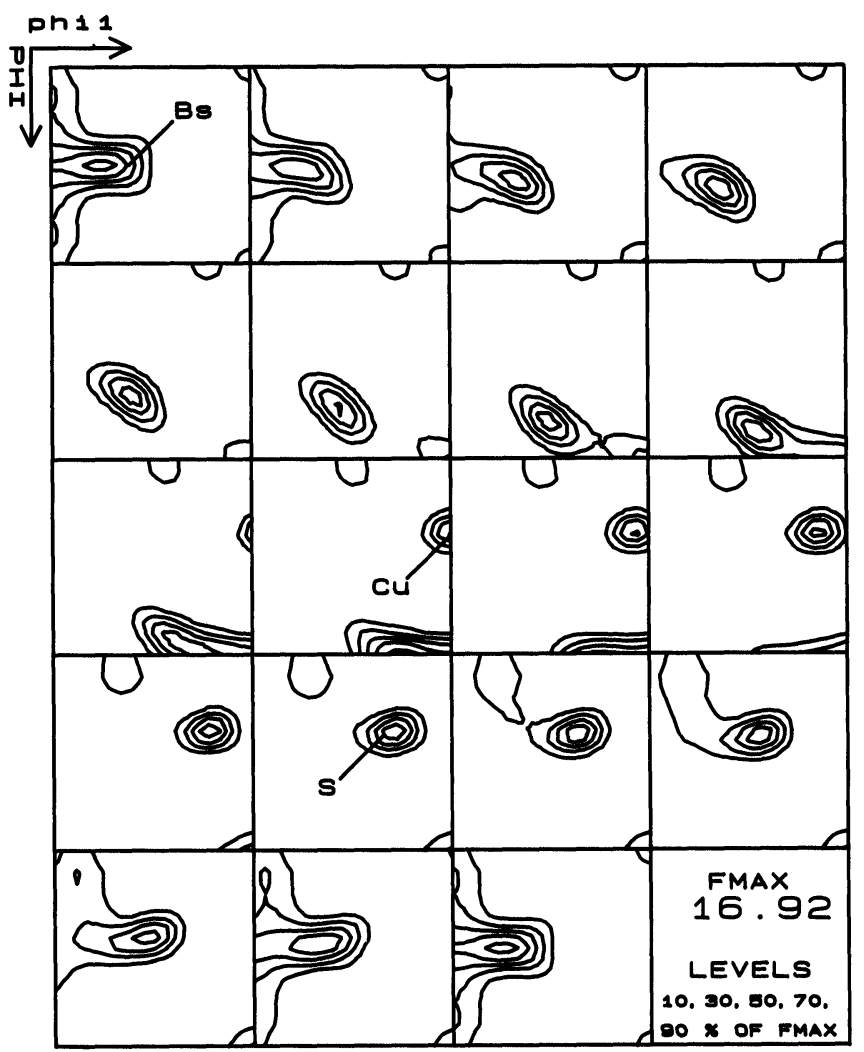

(c)

Figure 3 Simulated rolling texture ODFs from initial random texture, $\bar{\varepsilon}=2.4$ (a) $m=0.05, \zeta=0.1$, (b) $m=0.15, \zeta=0.25$, (c) $m=0.2, \zeta=0.5$.

Note also that increasing $m$ tends to decrease the fmax values; this is a well-known effect of high rate sensitivity values which tend to distribute the slip rate $\dot{\gamma}^{s}$ more uniformily, (Tóth et al. (1988)) so that the overall lattice rotation rates decrease leading to slightly weaker textures.

Another interesting feature of higher $m$ and $\zeta$ values is the influence on the stability of the Cube and Goss orientations, i.e. along the RD rotated cube fibre $\left(0<\phi<45^{\circ}\right)$. This is illustrated in Figure 7 by normalized $f(g) / \mathrm{fmax}$ plots for simulated textures using the 3 "temperature regimes." According to the model the stability of both the cube and Goss components should generally increase with increasing temperature.

\section{DISCUSSION}

A simple model has been presented to describe the influence of elevated temperatures on rolling texture formation of fcc metals. The model assumes that the rate sensitivity $m$ increases with temperature which is supported by most 

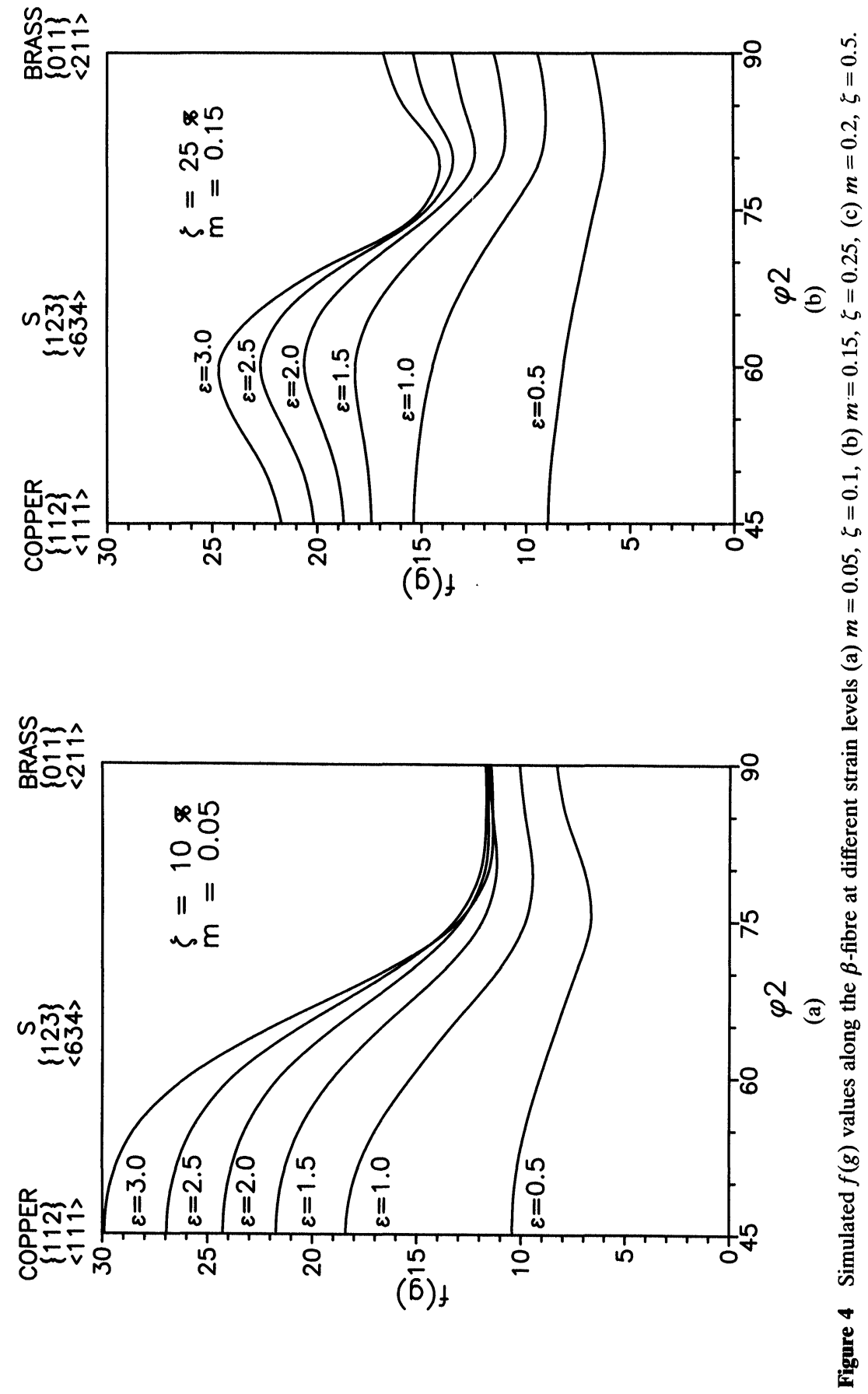


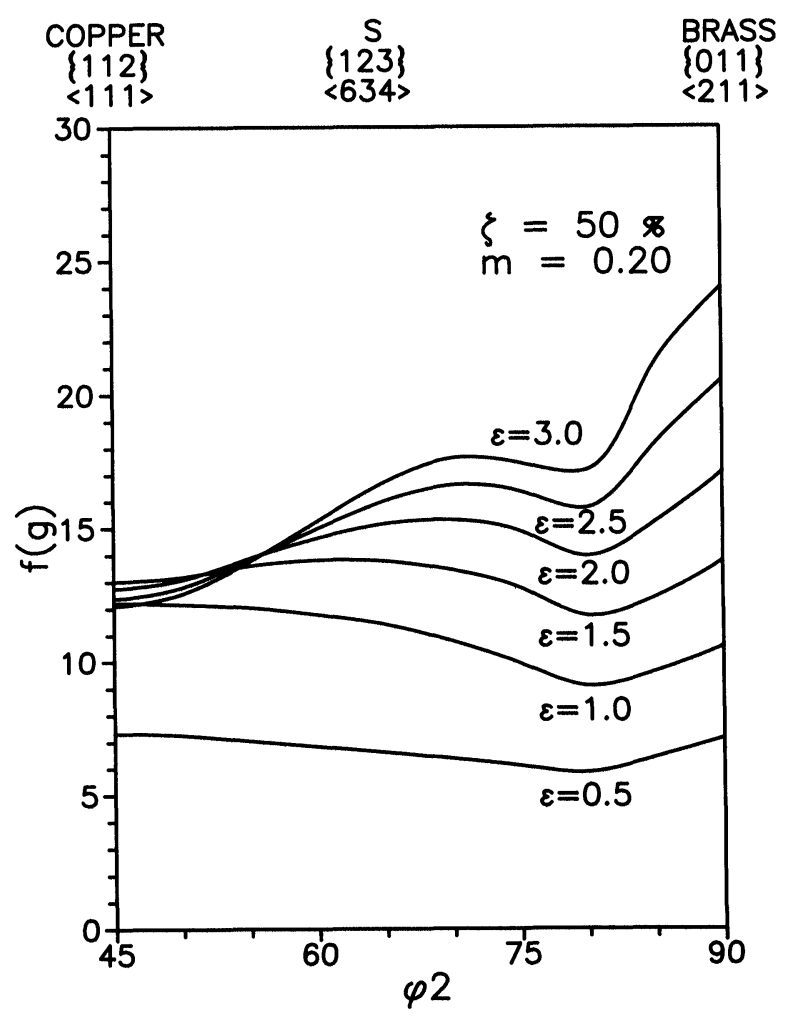

(c)

Figure 4 Simulated $f(g)$ values along the $\beta$-fibre at different strain levels (a) $m=0.05, \zeta=0.1$, (b) $m=0.15, \zeta=0.25$, (c) $m=0.2, \zeta=0.5$.

experimental data. However, as noted by Chen and Kocks (1991), the influence of the strain rate sensitivity by itself is insufficient to explain the large variations of rolling textures of most fcc alloys with increasing temperature. Our model further assumes that the amount of the $\varepsilon_{12}$ shear increases with temperature (although always significantly less that the value corresponding to the Sachs type RC model). This hypothesis is the most difficult to justify theoretically at the present time. There are good grounds for arguing that $\dot{\varepsilon}_{12}$ takes values between 0 and the Sachs type RC value $\dot{\varepsilon}_{12}^{0}$, as clearly shown by the channel die tests of partially constrained crystals. Other experimental measurements of grain shears in compressed $\mathrm{Al}$ polycrystals, even at room temperature, indicate significant $\varepsilon_{12}$ shears. Furthermore, as pointed out by Becker (1991), the stress states required to enforce $\dot{\varepsilon}_{12}=0$ for the near-Bs orientations are physically unreasonable; they require large $\sigma_{12}$ values of the order of the compressive stress. Some relaxation of $\dot{\varepsilon}_{12}$ is therefore inevitable. The problem is to quantify the degree of relaxation of grains in a rolled polycrystal as a function of temperature.

It is clear nevertheless that, using reasonable values of $m$ and $\zeta$, the model gives qualitatively good agreement with the high temperature rolling textures of aluminium. In particular, the model predicts an evolution of the $\beta$ fibre components from $\mathrm{Cu}$ to $\mathrm{Bs}+\mathrm{S}$ at high temperatures. 

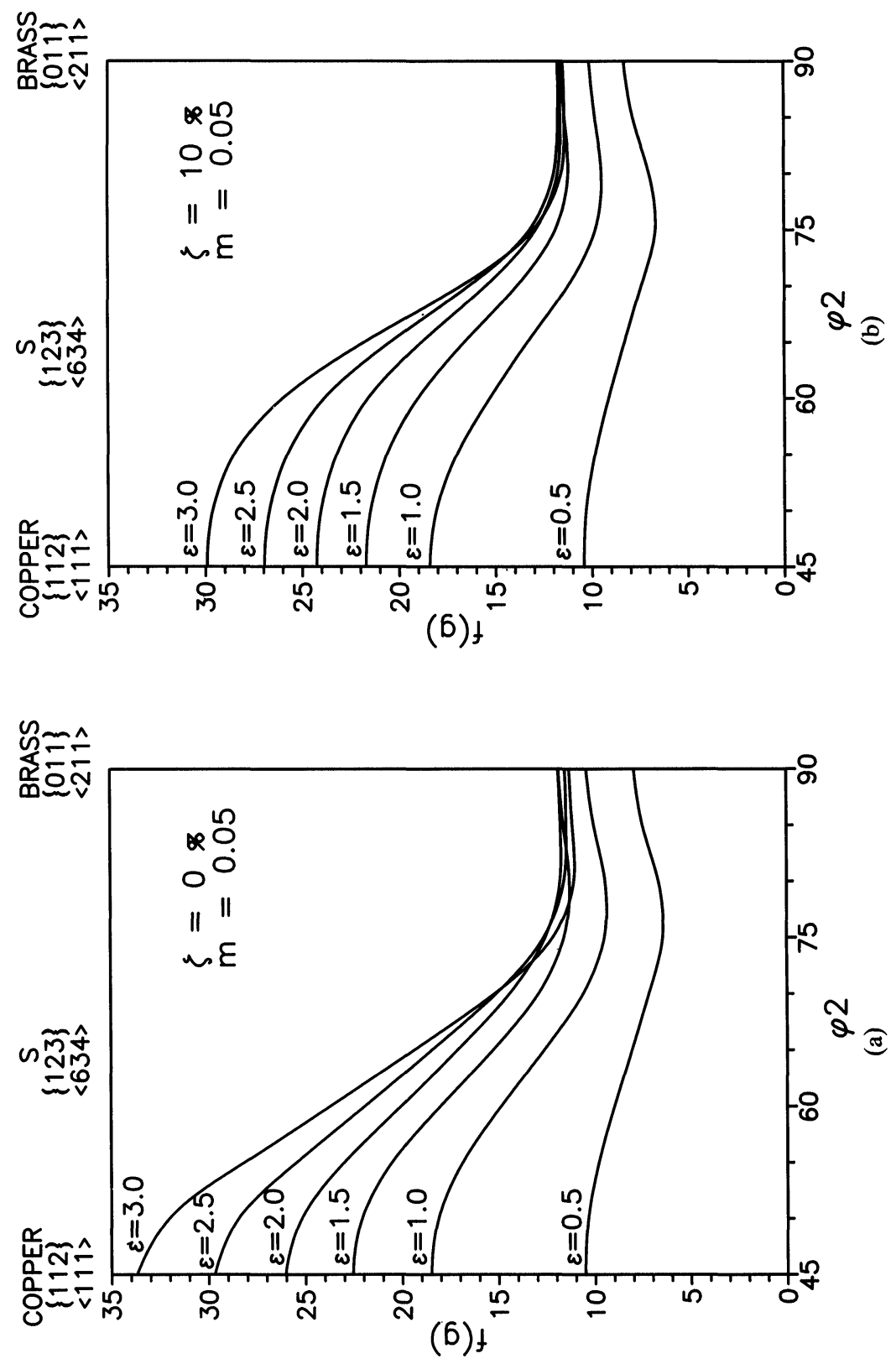


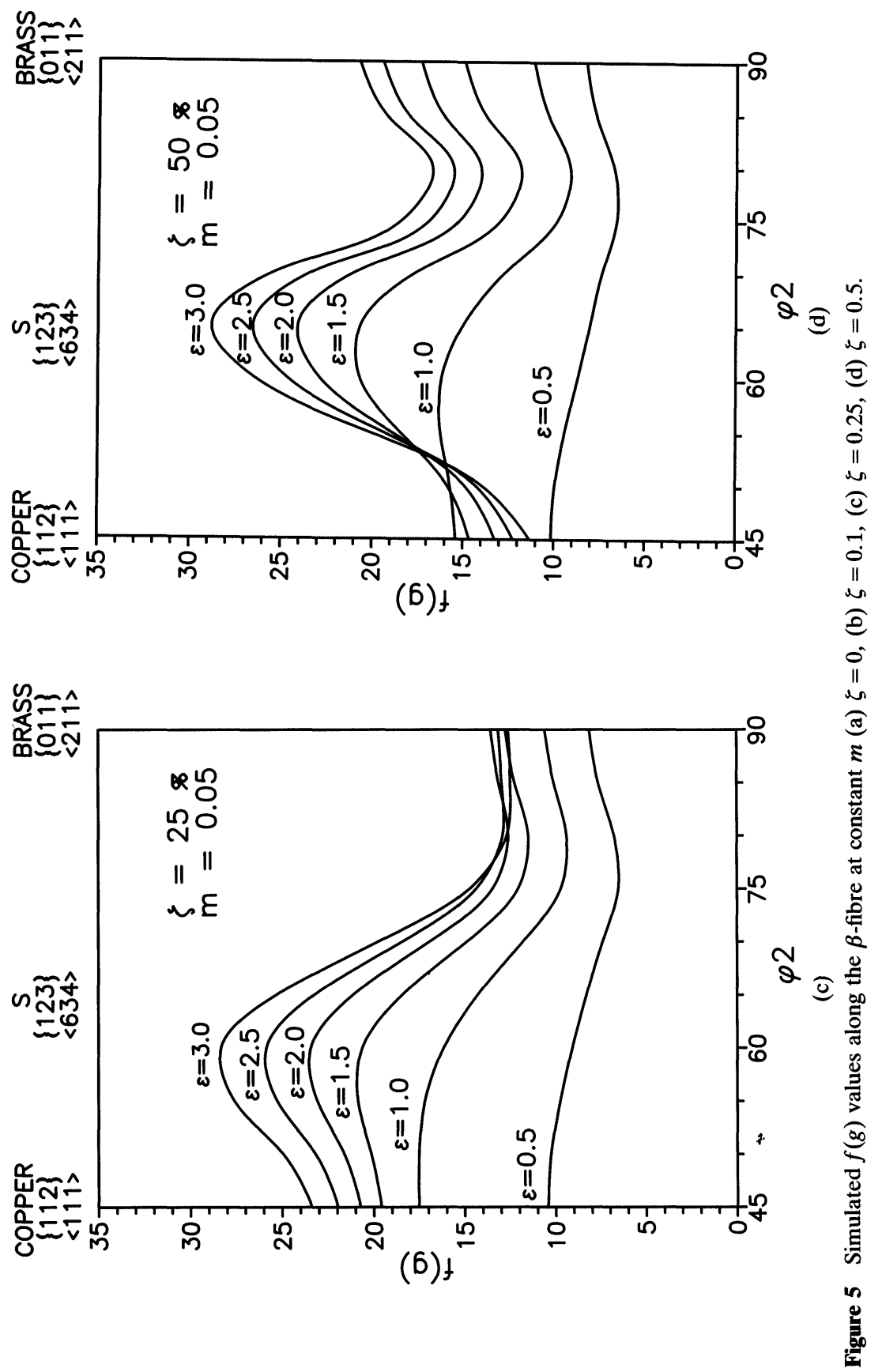



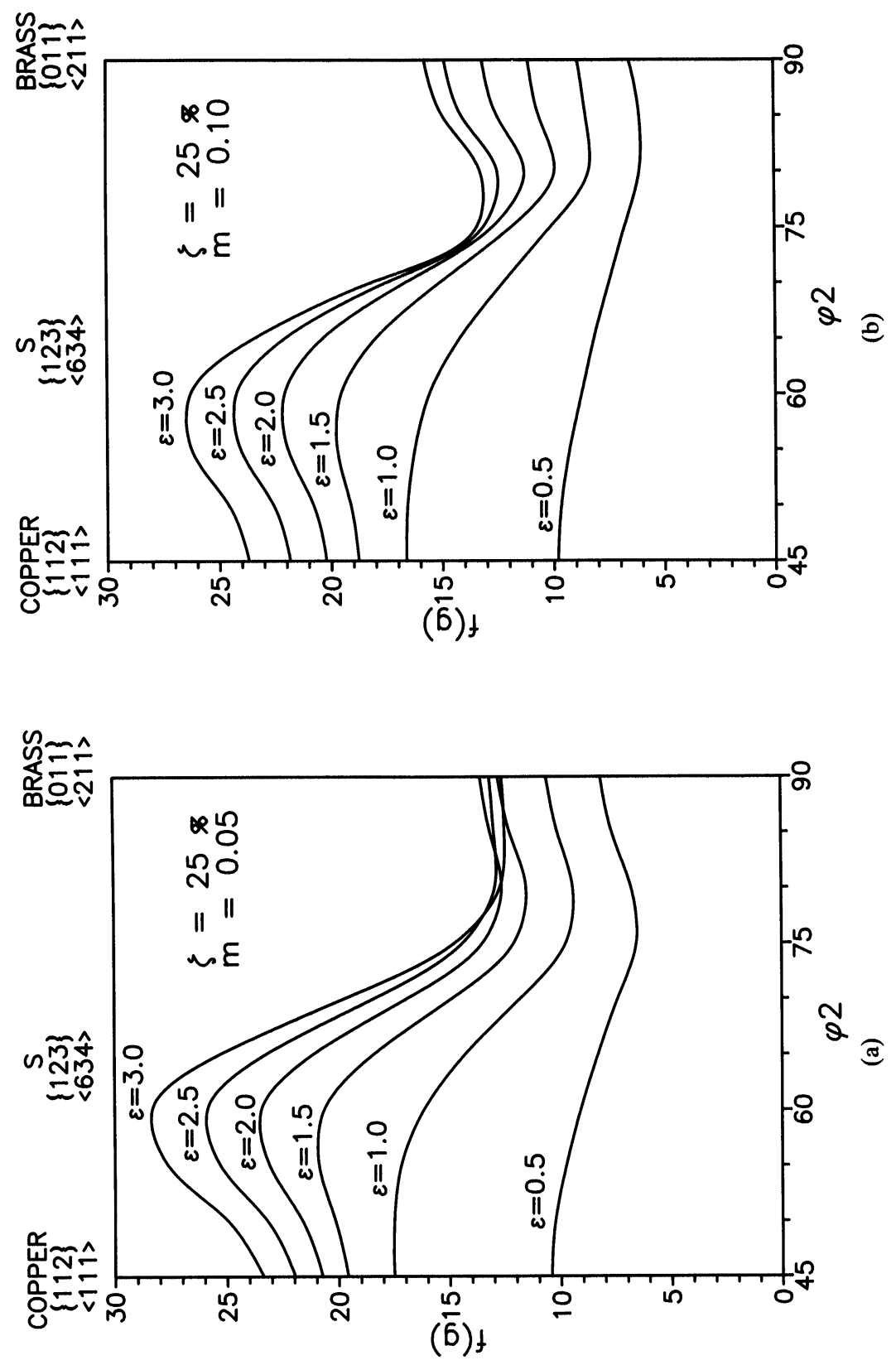


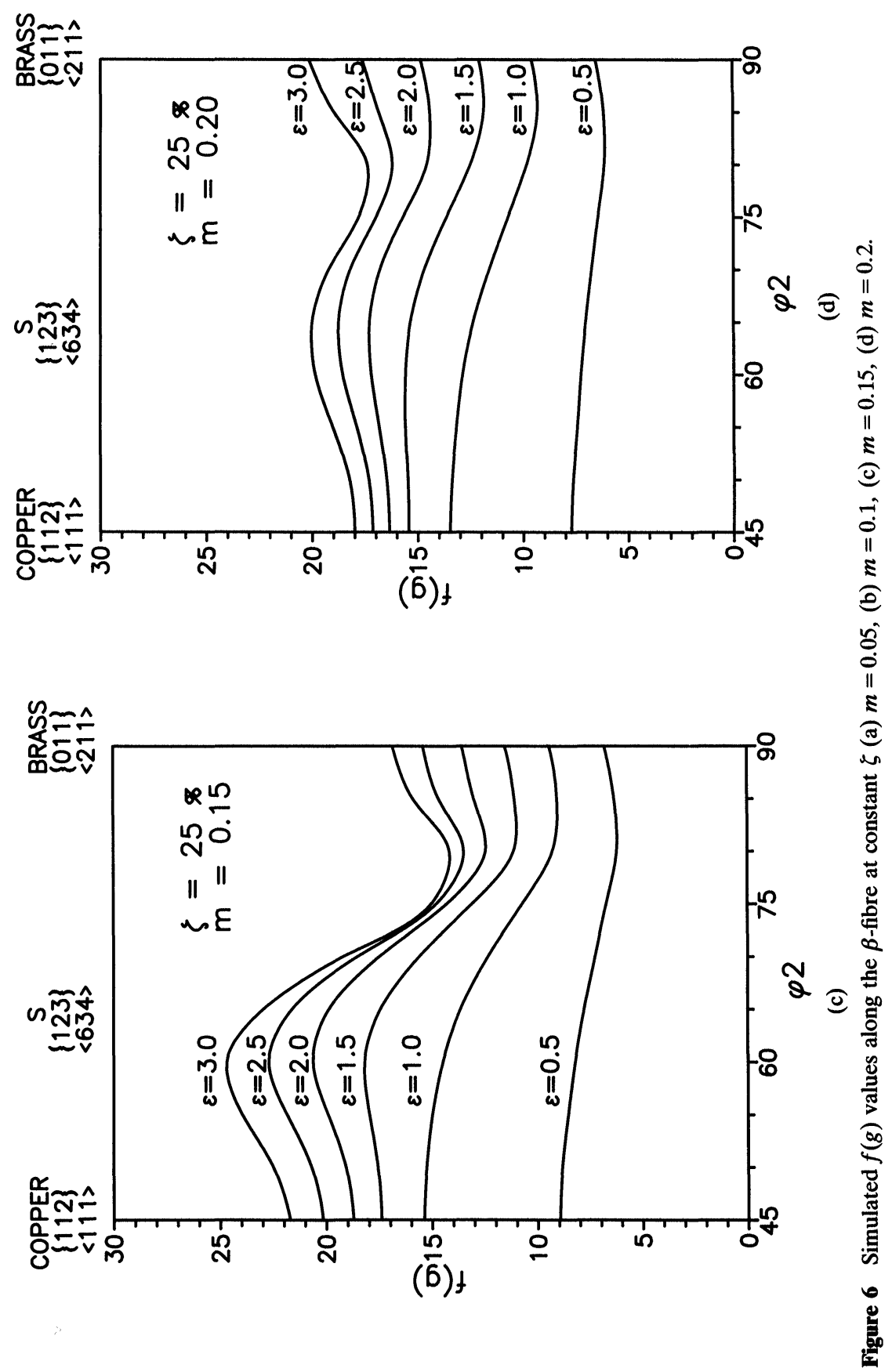




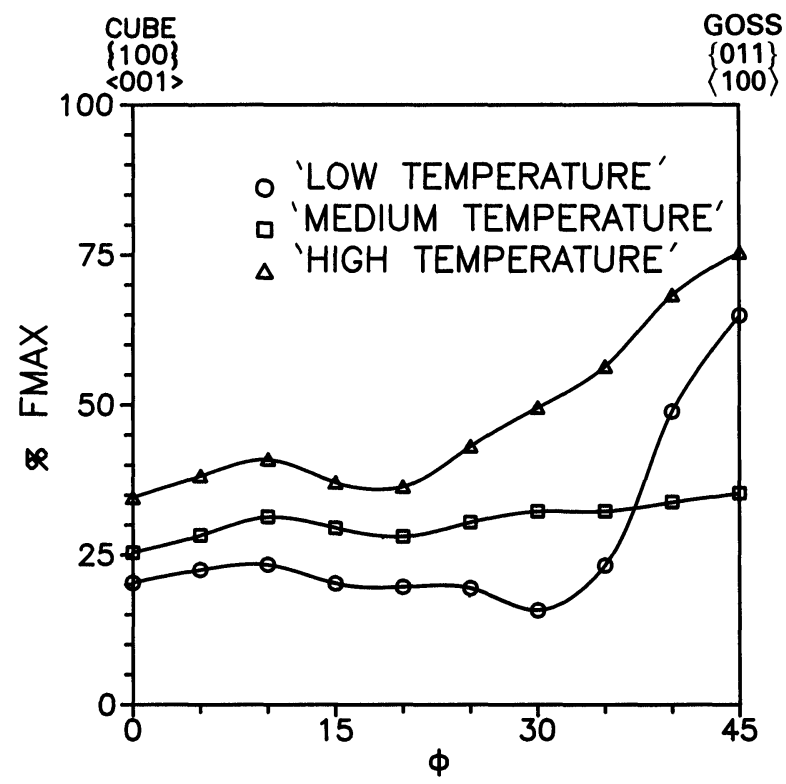

Figure 7 Relative orientation densities at $\varepsilon=1.5$ along the RD rotated cube fibre between Cube and Goss; "low temperature": $m=0.05, \zeta=0.1$; "medium temperature": $m=0.15, \zeta=0.25$; "high temperature": $m=0.2, \zeta=0.5$.

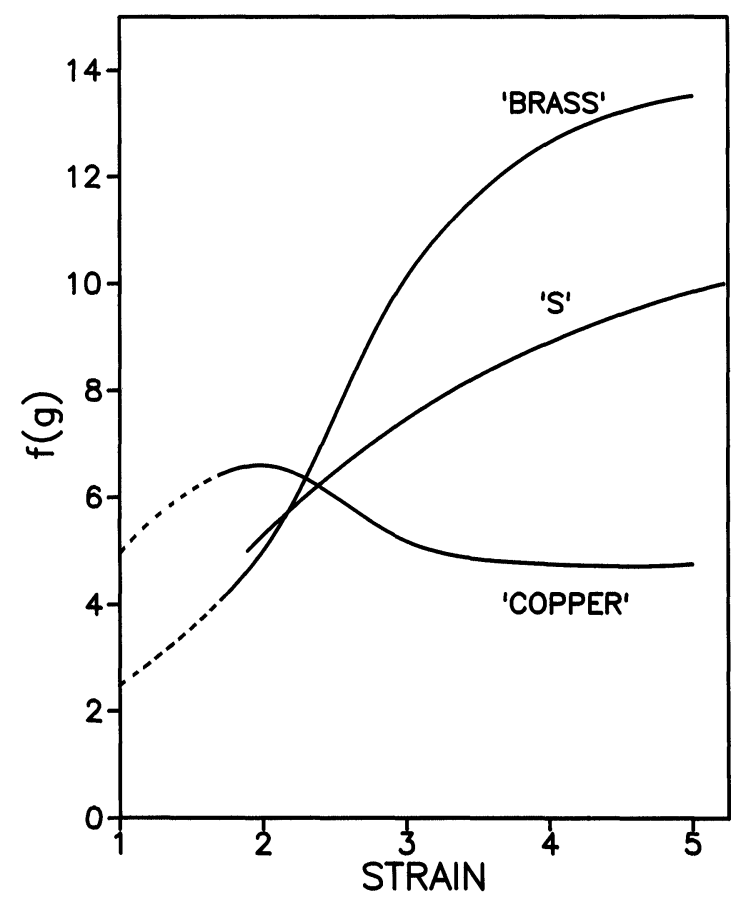

Figure 8 Experimental orientation densities of a 3104 alloy as a function of hot rolling strain. (after Bate and Oscarsson (1990)). 
Quantitative comparison of the model simulations with experimental hot rolling textures of fcc metals is hampered by the absence of good quantitative texture data. Most available data does not specify in sufficient detail the important parameters such as the initial texture, the rolling strain, strain rates or even temperature (since the latter vary during rolling). Qualitative comparisons do however show the trends predicted by the model. For example, most hot rolled high strength aluminium alloys (7000 series, $\mathrm{Al}-\mathrm{Li})$ develop, in the absence of recrystallization, very strong Bs textures (Hirsch (1990), Lücke et al. (1991)). Furthermore inspection of these data reveals that the $S$ component increases during hot rolling at the expense of the $\mathrm{Cu}$ component-as predicted by the present model. This is also confirmed by the ODFs of hot rolled Al-1\% Mn$1.2 \% \mathrm{Mg}$ (Bate and Oscarsson (1990)), shown in Figure 8. At a strain of 2, Cu, S and $\mathrm{Bs}$ have roughly similar densities (with a maximum near $\mathrm{Cu}$ ). At a strain of 5 both $\mathrm{S}$ and $\mathrm{Bs}$ have doubled in intensity and $\mathrm{Cu}$ has decreased slightly.

There is another useful feature of the present model predictions; both cube and Goss components should strengthen at higher rolling temperatures. Very recent work by Daaland et al. (1992) on a hot rolled 3104 aluminium alloy confirms this general trend.

\section{ACKNOWLEDGEMENTS}

Cl. Maurice thanks the Centre de Recherches de Voreppe (Pechiney Group) for the provision of a CIFRE Post-graduate grant. L. S. Tóth acknowledges the Ecole des Mines de Saint-Etienne for financial support during his stay. We are grateful to Prof. E. Nes (University of Trondheim) and Drs. G.-M. Raynaud and $\mathrm{R}$. Shahani (CRV) for many useful discussions and comments.

\section{References}

Bacroix, B. and Jonas, J. J. (1988a). Proc. ICOTOM 8, J. S. Kallend and G. Gottstein eds. Metal. Soc., pp 403-429.

Bacroix, B. and Jonas, J. J. (1988b). Textures and Microstructures 8 and 9, pp 267-311.

Bate, P. and Oscarsson, A. (1990). Mat. Sci. Tech. 6, pp 520-527.

Becker, R. (1991). J. Mech. Phys. Solids 39, pp 459-476.

Becker, R. and Lalli, L. A. (1991). Textures and Microstructures 14-18, pp 145-150.

Brown, B., Kim, K. H. and Anand, L. (1989). Int. J. Plasticity 5, pp 95-130.

Chen, S. R. and Kocks, U. F. (1991). Hot Deformation of Aluminium Alloys, Detroit, Michigan (1990), T. G. Longdon, H. D. Merchant, J. G. Morris and M. A. Zaidi eds. The Minerals, Metals and Materials Society, pp 89-104.

Daaland, O. et al. (1992). to be published in Proceedings ICAA3, Trondheim, 1992.

Fortunier, R. (1987). Doctoral Thesis, INP Grenoble-E. M. St Etienne.

Hirsch, J. (1990). Mat. Sci. Tech. 6, pp 1048-1057.

Hirsch, J. and Lucke, K. (1988). Acta Metall. 36, 2883.

Lucke, K., Engler, O., Driver, J. H., Delecroix, M. and Mizera, J. (1991). Brite-Euram Contract Report "Texture and Plastic Anisotropy in Aluminium-Lithium Alloys."

McGregor Tegart, W. J. (1966). Elements of Mechanical Metallurgy, Macmillan.

Maurice, Cl. (1992). Doctoral Thesis, INP Grenoble-E. M. St Etienne, (to be published).

Mizera, J. (1988). DEA, INP Grenoble-E. M. St Etienne.

Orlans-Joliet, B., Driver, J. H. and Montheillet, F. (1990). Acta Metall. Mater., 38, pp 581-594.

Pierce, D., Asaro, R. J. and Needleman, A. (1983). Acta Metall., 31, p 1951.

Tóth, L. S., Gilormini, P. and Jonas, J. J. (1988). Acta Metall., 36, p 3077.

T6th, L. S., Jonas, J. J. and Neale, K. W. (1990). Proc. Roy. Soc. Lond. A427, p 201.

Van Houtte, P. (1986). Proc. ICSMA 7, Montreal 1985, Vol III, H. J. McQueen, J. P. Bailon, J. L. Dickson, J. J. Jonas and M. G. Akben eds. Pergamon Press, Toronto, pp 1701-1725. 